\title{
RAMAN AND SURFACE ENHANCED RAMAN SIGNALS OF THE SENSOR 1-(4-MERCAPTOPHENYL)-2,4,6-TRIPHENYLPYRIDINIUM PERCHLORATE
}

\author{
FREDDY CELIS ${ }^{1,2 *}$, MARCELO CAMPOS-VALLETTE 3 , JOSÉ CÁRCAMO VEGA4, \\ JUAN S. GÓMEZ-JERIA AND CAROLINA ALIAGA ${ }^{5,6}$ \\ ${ }^{1}$ Centro de Estudios Avanzados (CEA), Universidad de Playa Ancha, Valparaiso, Chile. \\ ${ }_{2}^{2}$ Departamento de Química, Universidad de Playa Ancha, Valparaíso, Chile. \\ ${ }_{3}^{3}$ Departamento de Química, Facultad de Ciencias, Universidad de Chile. \\ ${ }^{4}$ Laboratorio de Análisis e Investigaciones Arqueométricas (LAIA), Departamento de Antropología, Universidad de Tarapacá, Arica, Chile. \\ ${ }^{5}$ Departamento de Ciencias del Ambiente, Universidad de Santiago de Chile. \\ ${ }^{6}$ Centro para el Desarrollo de la Nanociencia y la Nanotecnología, CEDENNA, Chile.
}

\begin{abstract}
The sensor 1-(4-mercaptophenyl)-2,4,6-triphenylpyridinium perchlorate compound was vibrationally characterized using Raman and the Surface-Enhanced Raman techniques, SERS and Shell-Isolated Nanoparticles-Enhanced Raman Spectroscopy (SHINERS). The Raman spectrum was analyzed and the band assignment was supported using DFT data at the B3LYP/6-31G(d) level. SERS data allowed infer about the orientation of the analyte on the naked Ag surface. EHT calculations for an Ag/analyte model represent well the SERS spectrum supporting the Ag-S bond formation. The SHINERS spectrum was obtained by using $\mathrm{Ag} @ \mathrm{SiO}_{2}$ nanoparticles prepared at two different time of the $\mathrm{SiO}_{2}$ coating process. The most intense SHINERS spectral signals of the compound (100 nM) were obtained after 20 minutes of the $\mathrm{Ag} @ \mathrm{SiO}_{2}$ formation. No charge-transfer was concluded from the SHINERS experiments.
\end{abstract}

Keywords: 1-(4-mercaptophenyl)-2,4,6-triphenylpyridinium perchlorate; Raman; SERS; SHINERS; DFT and EHT calculations.

\section{INTRODUCTION}

Pyridinium dyes are an interesting family of compounds due to their synthetic versatility and accessibility, being used as solvatochromic, thermohalochromic and halochromic sensors ${ }^{1-3}$. This compounds display poor fluorescent quantum yield; however, they have been employed to synthesize dual probes, spin and fluorescent, when the pyridinium unit is linked to a nitroxide moiety. These later probes have been employed to monitor $\mathrm{H}$-abstraction reactions $\mathrm{s}^{4}$. These compounds have been used as molecular bridges in electrochemical systems in order to reduce $\mathrm{O}_{2}^{5,6}$. Betaine-30 (5'-(2,4,6-triphenyl-1-pyridiniumyl)$1,1^{\prime}: 3^{\prime}, 1^{\prime}$ '-terphenyl-2'-olate) was studied using Raman data, supported by theoretical calculations ${ }^{7}$; other works shown the solvent effect on the Raman spectrum of this type of molecules ${ }^{8,9}$.

It is possible to enhance the emission and Raman scattering of a fluorophore, by using core@shell nanoparticles in the media under study; it is known that it is possible to enhance fluorescence signal, giving rise to the Shell-Isolated Nanoparticles-Enhanced Fluorescence (SHINEF) when the shell has a thickness around $5 \mathrm{~nm}^{10,11}$. With the same nanomaterial it is possible to enhance the Raman signal (SHINERS) ${ }^{12}$. In this case, the enhancement of the Raman signals depends on the thickness of the shell ( $2 \mathrm{~nm}$ approx.).

In this work, we assign and discuss for the first time the Raman and SERS spectra of 1-(4-mercaptophenyl)-2,4,6-triphenylpyridinium perchlorate (4-MPTP), a secondary betaine ${ }^{13}$, by using theoretical data. Calculations are performed using DFT data at the B3LYP/6-31G(d) level of the theory; the Extended Hückel theory is proposed to characterize the analyte surface interaction. Two different coating thickness of $\mathrm{SiO}_{2}$ on AgNPs are proposed for the SHINERS experiments.

\section{EXPERIMENTAL}

\section{Synthesis of 4-MPTP}

Chemicals for the synthesis of 4-MPTP, acetophenone, benzaldehyde, $\mathrm{HClO}_{4}$, 4-aminobenzenethiol, trisodium citrate, silver nitrate, (3-aminopropyl) trimethoxysilane and sodium silicate solution were purchased from Sigma and used without further purification. The synthesis was carried out in two steps. Step 1, synthesis of 2,4,6-triphenylpyrylium perchlorate: $2 \mathrm{mmol}$ of acetophenone were mixed with $1 \mathrm{mmol}$ of benzaldehyde at $120^{\circ} \mathrm{C}$, adding some drops of $\mathrm{HClO}$ and stirring during 30 minutes. The temperature was controlled at $20^{\circ} \mathrm{C}$ previous to add $3 \mathrm{~mL}$ of acetone and then precipitate the product in diethyl ether. The pyrylium salt obtained was washed with acetone and diethyl ether. $71 \%$ yield and m.p. $280^{\circ} \mathrm{C}$. Step 2 , synthesis of 1-(4-mercaptophenyl)2,4,6-triphenylpyridinium perchlorate: $5 \mathrm{mmol}$ of pyrylium salt were mixed with 7,4 mmol of 4-mercaptoaniline in ethanol; the mix was refluxed by 8 hours at $90^{\circ} \mathrm{C}$. The product was precipitated in diethyl ether and crystallized in ethanol. $65 \%$ yield and m.p. $290^{\circ} \mathrm{C} .{ }^{1} \mathrm{H}-\mathrm{NMR}$ (400 MHz, acetone-d6): $\delta 8,58$ $(\mathrm{s}, 2 \mathrm{H}), 7,58(\mathrm{~m}, 17 \mathrm{H}), 7,14(\mathrm{~d}, 2 \mathrm{H})$. The UV-VIS absorption and fluorescence emission spectra display maxima at $310 \mathrm{~nm}$ and $486 \mathrm{~nm}$, respectively.

\section{Preparation of $\mathrm{AgNPs}$ and $\mathrm{Ag} @ \mathrm{SiO}_{2}$}

The silver colloidal nanoparticles were prepared following a modified procedure by Lee and Meisel ${ }^{14} .9 \mathrm{mg}$ of silver nitrate are dissolved in $50 \mathrm{~mL}$ of milliQ water and heated to bring the solution to a boil. Once the solution brought up to a boil, the sodium citrate $(1 \%)$ is quickly added. Heating and stirring is maintained for $1 \mathrm{hr}$ after the addition of the sodium citrate. To 30 $\mathrm{mL}$ of AgNPs was added $0,4 \mathrm{~mL}$ of (3-aminopropyl)trimethoxysilane $1 \mathrm{mM}$ and stirring during 15 minutes. Then, $3,2 \mathrm{~mL}$ of silicate solution $(\mathrm{pH}=10)$ was added, stirring and heating at $90^{\circ} \mathrm{C}$. After 20 and 60 minutes the shellisolated nanoparticles (SHINs) of $\mathrm{Ag} @ \mathrm{SiO}_{2}$ were cleaned and concentrated by centrifugation (5 minutes at $5000 \mathrm{rpm}$ thrice). The $\mathrm{Ag} @ \mathrm{SiO}_{2}$ characterization was carried out using field emission SEM and AFM equipments. The dispersion of nanospheres was studied by AFM image. The results (Fig. 1A) indicate that the diameter of the $\mathrm{SiO}_{2}$ uncoated $\mathrm{Ag}$ nanospheres was around $50 \mathrm{~nm}$. The scanned area was $1 \mu \mathrm{m}^{2}$. The same image gives us a detailed dispersion of the AgNPs.

The methodology used to obtain the enhancement factor (EF) values in SERS and SHINERS spectra has been reported by Le Ru et al. ${ }^{15}$ and considers the intensity of any band present in both spectra $\left(\mathrm{EF}=\mathrm{I}_{\mathrm{SERS}} / \mathrm{I}_{\text {Raman }}\right)$.

\section{AgNPs and $\mathrm{Ag} @ \mathrm{SiO}_{2}$ characterization}

The plasmon signal of AgNPs and $\mathrm{Ag} @ \mathrm{SiO}_{2}$ was characterized using UVVIS spectroscopy. The AgNPs showed a maximum absorption at $405 \mathrm{~nm}$. The UV-VIS spectra of both $\mathrm{Ag} @ \mathrm{SiO}_{2}$ synthesized were shown an absorption band at $415 \mathrm{~nm}$. Field emission SEM showed two different thickness of $\mathrm{SiO}_{2}$ in coated AgNPs (Fig. 1B and 1C). The first thickness was estimated in $4 \mathrm{~nm}$ and the second in $8 \mathrm{~nm}$.

\section{Instrumentation}

Raman spectra were registered with a Renishaw micro-Raman system (RM1000) with the $785 \mathrm{~nm}$ laser line, a Leica microscope and an electrically cooled charge couple device (CCD) camera. The laser power on the fluorophore, fluorophore- $\mathrm{Ag}$ and the fluorophore- $\mathrm{Ag} @ \mathrm{SiO}_{2}$ systems was less than $2 \mathrm{~mW}$. The resolution was set at $4 \mathrm{~cm}^{-1}$. Spectra were recorded in the $200-3500 \mathrm{~cm}^{-1}$ region.

The AFM data were obtained by using a Nanoscope IIIa Extended Multimode AFM, Digital Instruments (Santa Barbara, CA, USA) with a "J" scanner. The surfaces were scanned in the Tapping ${ }^{\mathrm{R}}$ mode with a scan rate of $0.3 \mathrm{~Hz}$, using commercially etched silicon probes (Digital Instruments) with a 
triangular tip.

For the SEM measurements, samples of AgNPs dispersed in methanol were deposited onto carbon-coated copper grids. A JEOL JSM-7500F fieldemission scanning electron microscope equipped with a transmission electron detector (TED) was used to obtain low-resolution transmission images of the $\mathrm{Ag} @ \mathrm{SiO}$, nanoparticles.

NMR characterization was performed using a Bruker Avance-400 instrument operating at $400.13 \mathrm{MHz}$ for ${ }^{1} \mathrm{H}$. The solvent acetone- $\mathrm{d}_{6}$ was used at $25^{\circ} \mathrm{C}$.

The UV-VIS spectra of the 4-MPTP, AgNPs and Ag@SiO samples were acquired using the Lambda 1050 NIR-UV-VIS Perkin Elmer spectrophotometer. The fluorescence spectrum of 4-MPTP, was scanned using a Perkin Elmer LS-50 spectrometer.

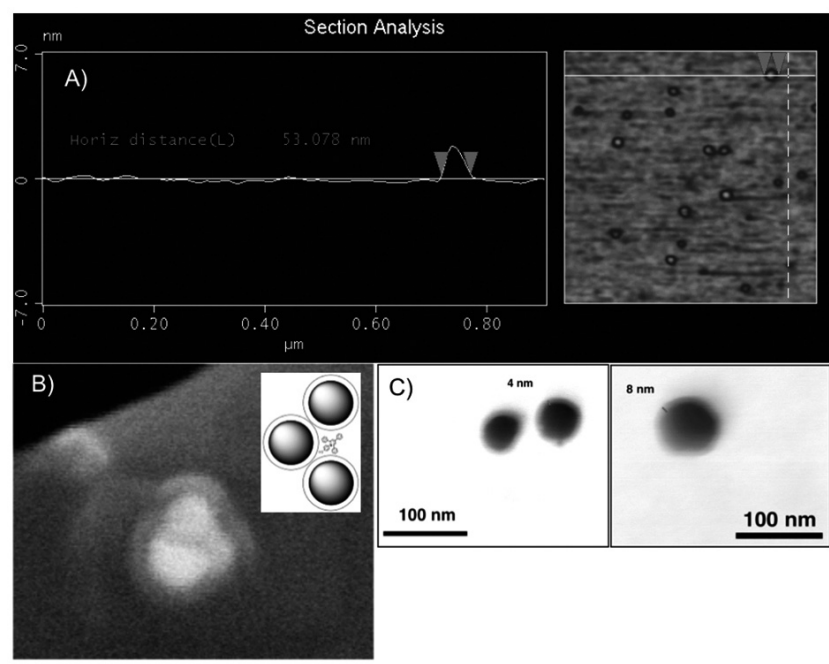

Figure 1. A) Transversal section analysis of $\mathrm{Ag} @ \mathrm{SiO}_{2} .50 \mathrm{~nm}$ of diameter of AgNPs. B) SEM image of $\mathrm{Ag} @ \mathrm{SiO}_{2}$ and scheme of cradle of hot-spots. C) SEM image of SHINs coated at 20 and 60 minutes.

\section{Calculation}

Calculations were performed with the Gaussian 03 package $^{16}$. The full geometry optimization of 4-MPTP was carried out at the B3LYP/6-31G(d) level of theory ${ }^{17-20}$.

The optimized structure was verified to correspond to a real minimum by establishing the absence of imaginary frequencies. By means of the FCART 01 software $^{21}$, the force constants obtained with Gaussian 03 were transformed to internal coordinates, allowing a compact representation and being naturally adapted to simple physical models.

A planar silver surface composed by 800 atoms was built as in our previous works ${ }^{22-25}$. In the first study, a 4-MPTP molecule was placed on the surface at different distances and orientations. A second study was carried out with the same Ag surface but with a 4-MPTP molecule bonded to an Ag atom at the center of the surface. The geometry optimization was carried out at the OPLS level by letting evolve the probe molecules over the rigid Ag surface. The Hyperchem software was used ${ }^{26,27}$. The $\mathrm{ClO}_{4}^{-}$counterion is not considered in the calculations.

\section{RESULTS AND DISCUSSION}

\section{A. The Raman spectrum}

The Raman spectrum of the solid 4-MPTP is displayed in Fig. 2A. The band assignment is performed on the basis of published data on related molecules $^{7}$, general characteristic group wavenumbers and the present calculations ${ }^{28,29}$. Figure 3 A shows the optimized geometry of 4-MPTP. Table 1 contains the calculated and experimental bond lengths. By comparison with the experimental geometrical parameters of 1,2,4,6-tetraphenylpyridinium ${ }^{30}$, the present bond lengths are confident; some variations, as a consequence of the steric bulk of the thiol moiety, are rather insignificants.

The Raman spectrum shows a weak band at $1633 \mathrm{~cm}^{-1}$ and a very strong band at $1610 \mathrm{~cm}^{-1}$, see Fig. 2A and Table 2. These bands are ascribed to coupled $v \mathrm{CC}$ and $v \mathrm{CN}$ aromatic modes. Band with medium intensity at 1509 $\mathrm{cm}^{-1}$ is assigned to $\delta \mathrm{CH}$ of the pyridinium ring. The strong Raman band at $1370 \mathrm{~cm}^{-1}$, was assigned to a $\delta \mathrm{CH}$ mode of the pyridinium ring coupled to an inter ring (B and C, Fig. 3B) vNC mode. The $1245 \mathrm{~cm}^{-1}$ band is ascribed to a $v \mathrm{CN}$ mode corresponding to the $\mathrm{CN}$ bond between the pyridinium and the mercaptophenyl ring. The strong band observed only in the Raman spectrum at $1005 \mathrm{~cm}^{-1}$ is ascribed to the phenyl ring (A, B') breathing mode. Others bands of the phenyl rings with weak intensities at 1195 and $1170 \mathrm{~cm}^{-1}$ in the Raman spectrum are assigned to the $\mathrm{CH}$ in-plane deformations. The weak band at 1035 $\mathrm{cm}^{-1}$ corresponds to a $\mathrm{CH}$ deformation and $\mathrm{vCC}$ modes of the mercaptophenyl ring. The medium weak bands at 845 and $674 \mathrm{~cm}^{-1}$ are assigned to the $\rho \mathrm{CH}$ mode; the last band is coupled to a $v$ CS mode. Bands at 401, 283, 246 and 234 $\mathrm{cm}^{-1}$ are ascribed to ring skeletal vibrations. The $v \mathrm{CS}$ vibration corresponds to the weak bands observed at 674 and $614 \mathrm{~cm}^{-1}$, the first coupled to $\rho \mathrm{CH}$ and the second to $\mathrm{vClO}_{4}^{-}$modes. Bands at 1080 and $937 \mathrm{~cm}^{-1}$ are attributed to stretching modes of the $\mathrm{ClO}_{4}^{-}$counterion; the band at $458 \mathrm{~cm}^{-1}$ is due to deformation modes of perchlorate coupled to a inter ring (B-C) $v N C$ mode. The medium broad band at $490 \mathrm{~cm}^{-1}$ probably contains information of vibrations belonging to the pyridinium ring involving the $\mathrm{N}$ atom. A disulfide bridge formation is not verified; the $\mathrm{VSS}$ mode for the ringC-S-S-ringC fragment should be expected in the $530-500 \mathrm{~cm}^{-1}$ region.
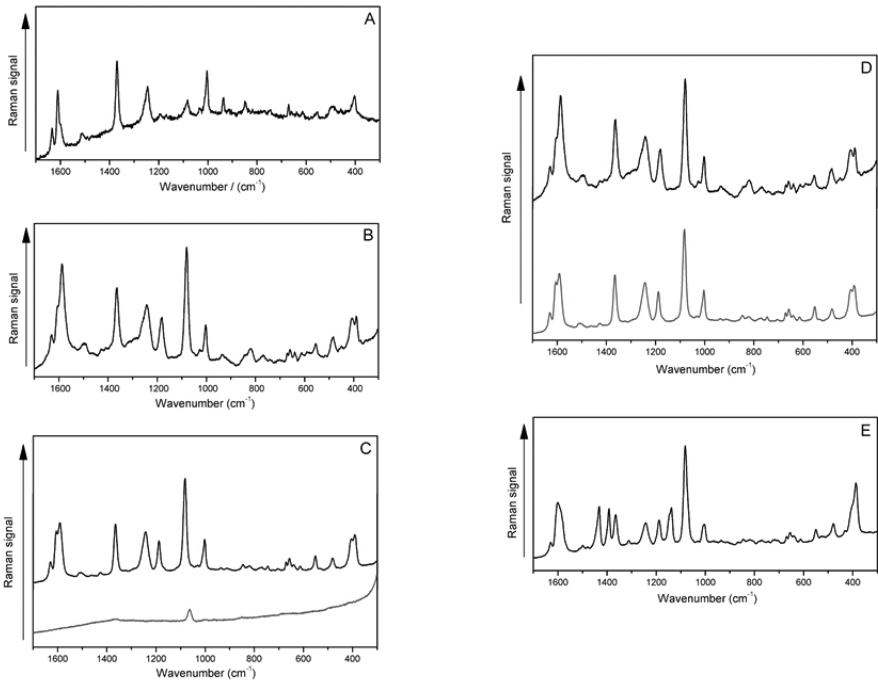

Figure 2. A) Raman spectrum of solid 4-MPTP. B) SERS spectrum of 4-MPTP $1 \mathrm{mM}$. C) Raman spectra of 4-MPTP $1 \mathrm{mM}$ with SHINs at 0.5 hours coating (top) and at 1 hour coating (bottom). D) SERS (top) and SHINERS (bottom) spectra of 4-MPTP $1 \mathrm{mM}$. E) SHINERS spectrum of 4-MPTP $1 \times 10^{-7}$ $\mathrm{M}(100 \mathrm{nM})$

\section{B. The SERS spectrum}

The relative intensity of several Raman bands of 4-MPTP is modified by the interaction with the Ag surface, see Fig. 2B. The wavenumber of some bands also changes. A perchlorate mode at $933 \mathrm{~cm}^{-1}$ is observed in the SERS spectrum $^{31}$. The $v \mathrm{Ag}-\mathrm{S}$ mode appears at $220 \mathrm{~cm}^{-132}$. These results suggest that 4-MTPT interacts with the metal surface. The intensity variation is associated to its molecular orientation on the surface. It is expected that the intensity of modes vibrating parallel to the laser excitation will be enhanced the most $t^{33}$. Thus, the first evidence of a perpendicular orientation of the 4-MTPT on the surface is the appearance of the $v \mathrm{Ag}-\mathrm{S}$ band. The relative intensity increasing of the bands ascribed to the $v C C$ mode of the B' rings at 1587 and $1080 \mathrm{~cm}$ 1 , the most enhanced in the SERS spectrum, could be due to a rather tilted position of the lateral phenyl rings, respect to the surface. The band at 1080 $\mathrm{cm}^{-1}$ belongs to a mode coupled to a $\mathrm{vCC}$ mode of the B ring; this suggests that the corresponding ring is perpendicular to the surface. The increasing of the relative intensity of the $\delta \mathrm{CH}$ modes belonging to the lateral phenyl rings at $1179 \mathrm{~cm}^{-1}$ supports the idea of a tilted orientation of such fragments respect to the surface. The intensity of the band at $1363 \mathrm{~cm}^{-1}$ also increases by surface effect, however the enhancement is rather feeble. The vibrational modes involved in this band are the in plane deformation of $\mathrm{CH}$ of the pyridinium ring and an interring $v \mathrm{NC}$ modes. Then, it is expected that the $\mathrm{CN}$ inter ring bond could be perpendicular to the surface. By assuming that the molecular rings are oriented perpendicular or tilted to the surface, the proposed assignment of the enhanced band at $819 \mathrm{~cm}^{-1}$ to a $\rho \mathrm{CH}$ ringC, should not be adequate. This large 
band should contain information about a vibration involving the CS bond. The band in the SERS spectrum corresponding to the coupled $v \mathrm{CS} / \mathrm{vClO}{ }_{4}^{-}$mode at 610 $\mathrm{cm}^{-1}$ is enhanced by surface effect. If the whole molecule is oriented perpendicular to the surface, the $764 \mathrm{~cm}^{-1}$ band only observed in SERS could be ascribed to a $\delta \mathrm{CH}$ mode of the mercaptophenyl or pyridinium rings. A brief assignment of the SERS bands is shown in Table 3.

Table 1. Selected B3LYP/6-31G(d) geometrical parameters for 4-MPTP and experimental reported data ${ }^{30}$.

\begin{tabular}{|c|c|c|c|c|c|}
\hline Bonds & B3LYP/6-31G(d) & Exp. Data Ref 30. & Bonds & B3LYP/6-31G(d) & Exp. Data Ref 30. \\
\hline \multicolumn{3}{|c|}{ Ring A Bond lengths ( $(\AA)$} & \multicolumn{3}{|c|}{ Ring B Bond lengths ( $(\AA)$} \\
\hline $\mathrm{C} 1-\mathrm{C} 2$ & 1.3971 & 1.35 & $\mathrm{C} 12-\mathrm{C} 13$ & 1.4047 & 1.41 \\
\hline $\mathrm{C} 2-\mathrm{C} 3$ & 1.3916 & 1.38 & $\mathrm{C} 12-\mathrm{C} 14$ & 1.4047 & 1.38 \\
\hline $\mathrm{C} 3-\mathrm{C} 4$ & 1.4081 & 1.36 & $\mathrm{C} 13-\mathrm{C} 15$ & 1.3869 & 1.37 \\
\hline $\mathrm{C} 4-\mathrm{C} 5$ & 1.4081 & 1.36 & $\mathrm{C} 14-\mathrm{C} 17$ & 1.3869 & 1.37 \\
\hline $\mathrm{C} 5-\mathrm{C} 6$ & 1.3916 & 1.38 & $\mathrm{C} 17-\mathrm{N}$ & 1.3814 & 1.38 \\
\hline $\mathrm{C} 6-\mathrm{C} 1$ & 1.3971 & 1.32 & $\mathrm{C} 15-\mathrm{N}$ & 1.3814 & 1.38 \\
\hline \multicolumn{3}{|c|}{ Ring C Bond lengths ( $(\AA)$} & \multicolumn{3}{|c|}{ Bond lengths ( $(\AA)$} \\
\hline $\mathrm{C} 19-\mathrm{C} 20$ & 1.3949 & 1.39 & $\mathrm{C} 4-\mathrm{C} 12$ & 1.4722 & 1.47 \\
\hline $\mathrm{C} 20-\mathrm{C} 22$ & 1.3900 & 1.36 & $\mathrm{C} 15-\mathrm{C} 32$ & 1.4862 & 1.48 \\
\hline $\mathrm{C} 22-\mathrm{C} 26$ & 1.4052 & 1.34 & $\mathrm{C} 17-\mathrm{C} 43$ & 1.4861 & 1.46 \\
\hline $\mathrm{C} 24-\mathrm{C} 26$ & 1.4035 & 1.35 & $\mathrm{C} 19-\mathrm{N}$ & 1.4603 & 1.46 \\
\hline $\mathrm{C} 24-\mathrm{C} 21$ & 1.3920 & 1.41 & & & \\
\hline C21-C19 & 1.3935 & 1.38 & & & \\
\hline
\end{tabular}

\section{Theoretical aspects of the Ag-4MPTP molecular model}

The final geometry of 4-MPTP interacting with the Ag surface is shown in Fig. 3C. If this is the actual position of the molecule during a SERS experiment (and if it is located on a hot spot), the SERS spectra should shown enhancement of bands assigned for instance, to the $\mathrm{CN}$ inter ring stretching mode; the SERS spectra results and the SERS selection rules show that this is not the case ${ }^{33}$. The final geometry of 4-MPTP bonded to the Ag surface through the thiol fragment is shown in Fig. 3D. It can be observed that the 4-MPTP molecule is proposed to be deprotonated, which is supported on the fact that the thiol moiety is highly reactive to a metal surface ${ }^{34}$. The calculated $\mathrm{Ag}$-S distance is $2.4 \AA$, which is quite similar to the observed value ${ }^{35}$.to correlate the nitrile stretching frequency with the local electric field exploiting the vibrational Stark effect (VSE This model predicts, following the SERS selection rules ${ }^{33}$, that the $v C S\left(638 \mathrm{~cm}^{-1}\right)$ and ring breathing $\left(1080 \mathrm{~cm}^{-1}\right)$ modes should be enhanced. The analysis of the SERS spectrum of Fig.2B shows that this model explains well the experimental results, thus proposing a rather tilted orientation of the molecule on the surface.

\section{The SHINERS spectrum}

Two different thicknesses of $\mathrm{SiO}_{2}$ were used to obtain the SHINERS spectra. (Fig. 2C).

$\mathrm{SiO}$, thickness: $2 \mathrm{~nm}$. A SHINERS effect is inferred from the spectrum obtained by using the coated Ag nanoparticles and 4-MPTP $10^{-3} \mathrm{M}$. The SHINERS spectral profile is not different to the SERS one; the general spectral intensity for the same analyte concentration and identical spectral scanning conditions is higher in SHINERS than in SERS, see Fig. 2D. The increase in the intensity is related to the existence of a "cradle of hot-spots" resulting from the synthesis of the shell-isolated nanoparticles (Fig. 1B), described by Aroca et al..$^{36}$ as aggregated nanoparticles. From the SHINERS data it is then possible an additional enhancement of the SERS weak modes (Table 3). Thus, SHINERS spectra allow reduce the detection limits of an analyte, as determined for 4-MPTP $100 \mathrm{nM}$, see Fig. 2E. The EF values indicate that the best surface to study this kind of compounds is $\mathrm{Ag} @ \mathrm{SiO}_{2}$, because it is possible to obtain a spectrum at very low concentration with an appropriate intensity. The EF value for the SERS band at $1002 \mathrm{~cm}^{-1}$ is 1,8 : the EF value for the SHINERS at $10^{-3} \mathrm{M}$ is 6,9 and for SHINERS at $10^{-7} \mathrm{M}$ is 3,86 .

Silica thickness: $4 \mathrm{~nm}$. By increasing the coating thickness the SHINERS effect is poor relative to the use of $2 \mathrm{~nm}$ silica thickness, Fig. 2C. In spite of the coating increasing it is still possible to observe Raman signals. In fact, only two bands ascribed to the silica coating are now observed at 1366 and 1062 $\mathrm{cm}^{-1}$. Thus, the Raman signals enhancement is delimited by the thickness of the coating, which is in good agreement with that already proposed by Tian et al. ${ }^{12}$ comparable to single-molecule fuorescence spectroscopy. However, generally substrates based on metals such as $\mathrm{Ag}$, $\mathrm{Au}$ and $\mathrm{Cu}$, either with roughened surfaces or in the form of nanoparticles, are required to realise a substantial SERS effect, and this has severely limited the breadth of practical applications of SERS. A number of approaches have extended the technique to non-traditional substrates, most notably tip-enhanced Raman spectroscopy (TERS

The main role of silica shell around the silver nanoparticle is to avoid the metal-ligand complex formation. Therefore, the chemical contribution to the signal enhancement due to the 4-MPTP/AgNP interaction is discarded. The electromagnetic contribution to the spectral enhancement is verified, and the intensity arises from the proximity of the analyte to the silver surface.

\section{CONCLUSIONS}

The Raman spectrum of 4-MPTP was obtained; the band assignment was supported by using DFT theoretical data at the B3LYP/6-31G(d) level. The SERS spectrum of the analyte was obtained and assigned on the basis of the Raman data. SERS data allowed infer that the analyte is oriented tilted on the Ag surface. Theoretical calculation carried out for the 4-MPTP molecule interacting with the $\mathrm{Ag}$ surface explains quite well the SERS spectrum; a formal Ag-S bond is concluded. Enhanced Raman signals were also obtained by using $\mathrm{Ag} @ \mathrm{SiO}_{2}$ nanoparticles; this enhancement resulted to be 5.1 times relative to that obtained in SERS. The SHINERS spectrum is verified without a particular organization of the compound on the coated metal surface. Finally, the present SHINERS data should be useful to study the hydrogen radical abstraction reaction at very low concentrations.

\section{ACKNOWLEDGEMENTS}

FC acknowledges the CONICYT scholarship Ph.D., AT 24121324-CONICYT support and the laboratory of Professor M. Caroli Rezende of the Universidad de Santiago de Chile, for facilities to perform the synthesis of 4-MPTP. JCV acknowledge Convenio de Desempeño Universidad de Tarapacá-Mineduc Humanidades, Artes y Ciencias Sociales (2013-2016). This work was financially supported by projects Fondecyt 1110736 and 1110106. 
Table 2. Experimental and calculated wavenumbers $\left(\mathrm{cm}^{-1}\right)$ and bands assignment.

\begin{tabular}{|c|c|c|c|}
\hline Raman & DFT & FCART & Assignment \\
\hline- & 2704 & 2594 & vSH \\
\hline $1633 \mathrm{~m}$ & 1650 & 1599 & $\nu \mathrm{CC}$ ring $\mathrm{A}, \mathrm{B}, \mathrm{C}+\nu \mathrm{CN}$ ring $\mathrm{B}$ \\
\hline $1610 \mathrm{vs}$ & 1644 & 1593 & $\nu \mathrm{CC}$ ring $\mathrm{C}+\nu \mathrm{CN}$ ringB \\
\hline $1598 \mathrm{sh}$ & 1631 & 1580 & $v^{\prime} C$ ringB' \\
\hline $1509 w$ & 1574 & 1527 & $\delta \mathrm{CH}$ ringB \\
\hline 1370vs & 1379 & 1340 & $\delta \mathrm{CH}$ ringB $+v \mathrm{NC}$ ringB-C \\
\hline $1245 \mathrm{~s}$ & 1264 & 1226 & $\mathrm{vCN}$ ringB-ring $\mathrm{C}$ \\
\hline $1195 \mathrm{w}$ & 1224 & 1189 & $\delta \mathrm{CH}$ ring $\mathrm{A}, \mathrm{B}$ \\
\hline $1170 \mathrm{w}$ & 1218 & 1184 & $\delta \mathrm{CH}$ ring $\mathrm{A}, \mathrm{B}^{\prime}$ \\
\hline $1080 \mathrm{~m}$ & 1108 & 1080 & $v \mathrm{CC}$ ring $\mathrm{B}, \mathrm{B}^{\prime}+v \mathrm{ClO}_{4}^{-}$ \\
\hline $1035 w$ & 1045 & 1022 & $\delta \mathrm{CH}$ ring $\mathrm{C}+v \mathrm{CC}$ ring $\mathrm{C}$ \\
\hline $1005 \mathrm{~s}$ & 1016 & 1000 & Ring breathing A,B' \\
\hline $937 \mathrm{~m}$ & - & - & $\mathrm{vClO}_{4}^{-}$ \\
\hline $845 \mathrm{~m}$ & 855 & 836 & $\rho \mathrm{CH}$ ring $\mathrm{A}$ \\
\hline $817 \mathrm{vw}$ & 829 & 815 & $\rho \mathrm{CH}$ ringC \\
\hline $745 w$ & 758 & 746 & $\rho \mathrm{CH}$ ringC $+v \mathrm{CS}$ \\
\hline $674 \mathrm{~m}$ & 667 & 655 & $\rho \mathrm{CH}$ ring $\mathrm{B}^{\prime}+v \mathrm{CS}$ \\
\hline $636 \mathrm{w}$ & 657 & 645 & $\rho \mathrm{CH}$ ring $\mathrm{C}+v \mathrm{C}-\mathrm{S}$ \\
\hline $614 \mathrm{w}$ & 625 & 616 & $v \mathrm{CS}+v \mathrm{ClO}_{4}^{-}$ \\
\hline $554 w$ & 537 & 528 & $\rho \mathrm{CH}$ ring $\mathrm{C}$ \\
\hline $490 \mathrm{~m}$ & 504 & 495 & $\rho \mathrm{CH}$ ring $\mathrm{B}$ \\
\hline $458 w$ & 483 & 475 & $\mathrm{ClO}_{4}^{-}$def $+v \mathrm{NC}$ (ringB-C) \\
\hline $401 \mathrm{~m}$ & 412 & 403 & Skeletal def out of plane (ringA,B') \\
\hline $283 w$ & 290 & 285 & Skeletal def. (ringB,B') \\
\hline 246w & 243 & 238 & Skeletal def. (ringA,B,B') \\
\hline $234 \mathrm{w}$ & 234 & 228 & Skeletal stretch. (ringA,B,C) \\
\hline
\end{tabular}

Symbols and abbreviations: $v$ stretching, $\delta$ in plane bending, $\rho$ out-of-plane bending, def. deformation, s strong, vs very strong, w weak, vw very weak, sh shoulder.

Table 3. Selected bands $\left(\mathrm{cm}^{-1}\right)$ in Raman, SERS and SHINERS.

\begin{tabular}{|c|c|c|c|}
\hline $\begin{array}{c}\text { Raman } \\
\text { band }\left(\mathrm{cm}^{-1}\right)\end{array}$ & $\begin{array}{l}\text { SERS band } \\
\left(\mathrm{cm}^{-1}\right)\end{array}$ & $\begin{array}{l}\text { SHINERS } \\
\text { band }\left(\mathrm{cm}^{-1}\right)\end{array}$ & Assignment \\
\hline $1633 \mathrm{~m}$ & $1630 \mathrm{~s}$ & $1630 \mathrm{~s}$ & $\nu \mathrm{CC}$ ring $\mathrm{A}, \mathrm{B}, \mathrm{C}+\nu \mathrm{CN}$ ring $\mathrm{B}$ \\
\hline $1610 \mathrm{vs}$ & $1605 \mathrm{sh}$ & $1605 \mathrm{sh}$ & $v \mathrm{CC}$ ring $\mathrm{C}+v_{\mathrm{CN}} \operatorname{ringB}$ \\
\hline $1598 \mathrm{sh}$ & $1587 \mathrm{vs}$ & $1590 \mathrm{vs}$ & ${ }^{\prime} \mathrm{CC}$ ringB' \\
\hline $1509 w$ & $1496 w$ & $1505 \mathrm{w}$ & $\delta \mathrm{CH}$ ringB \\
\hline $1370 \mathrm{vs}$ & $1363 \mathrm{~s}$ & $1366 \mathrm{vs}$ & $\delta \mathrm{CH}$ ringB $+v \mathrm{NC}$ ringB-C \\
\hline $1170 w$ & $1179 \mathrm{~s}$ & $1189 \mathrm{~s}$ & $\delta \mathrm{CH}$ ring $\mathrm{A}, \mathrm{B}^{\prime}$ \\
\hline $1080 \mathrm{~m}$ & 1080vs & $1082 \mathrm{vs}$ & $v \mathrm{CC}$ ring $\mathrm{B}, \mathrm{B}^{`}+v \mathrm{ClO}_{4}^{-}$ \\
\hline $1005 \mathrm{~s}$ & $1002 \mathrm{~m}$ & $1002 \mathrm{~s}$ & Ring breathing A,B' \\
\hline- & $933 w$ & - & $\mathrm{vClO}_{4}^{-}$ \\
\hline $817 \mathrm{vw}$ & $819 \mathrm{~m}$ & $821 w$ & $\rho \mathrm{CH}$ ringC \\
\hline- & $764 w$ & $767 w$ & $\rho \mathrm{CH}$ ringB or ringC \\
\hline $636 w$ & $638 w$ & $640 w$ & $v \mathrm{C}-\mathrm{S}$ \\
\hline $614 w$ & $610 w$ & $613 w$ & $v \mathrm{CS}+v \mathrm{ClO}_{4}^{-}$ \\
\hline- & $220 v s$ & - & $v A g S$ \\
\hline
\end{tabular}

A)

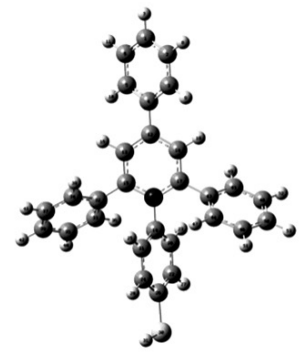

C)

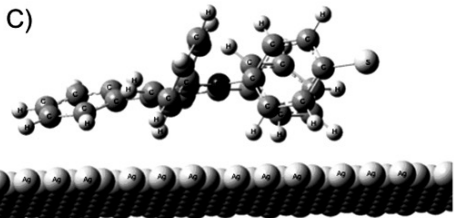

Figure 3. A) Optimized molecular model structure of 4MPTP and B) general structure. C) 4-MPTP not bonded to a Ag surface and D) Ag - 4-MPTP bonded ( $2.42 \AA)$.

\section{REFERENCES}

1. C. Reichardt. Chem. Soc. Rev., 21(3), 147-153, (1992).

2. M. Domínguez and M. Rezende. J. Iran. Chem. Soc., 7(4), 995-1003, (2010).

3. M. C. Rezende and A. Aracena. Spectrochim. Acta. A. Mol. Biomol. Spectrosc., 98, 18-22, (2012).

4. C. Aliaga, M. C. Rezende, and C. Tirapegui. Tetrahedron, 65(31), 6025 6028, (2009)

5. I. Ponce, J. Silva, R. Oñate, M. Rezende, M. Paez, J. Zagal, F. Mendizabal, J. Pavez, M. Miranda-Rojas, A. Munoz-Castro, and R. Arratia-Perez. $J$. Phys. Chem. C., 116(29), 15329-15341, (2012).

6. I. Ponce, J. F. Silva, R. Oñate, M. C. Rezende, M. a. Páez, J. Pavez, and J. H. Zagal. Electrochem. Commun., 13(11), 1182-1185, (2011).

7. S. Hogiu, J. Dreyer, M. Pfeiffer, K. Brzezinka, and W. Werncke. J. Raman Spectrosc., 31(8-9), 797-803, (2000).

8. X. Zhao, J. a Burt, and J. L. McHale. J. Chem. Phys., 121(22), $11195-$ 11201, (2004).

9. Y. Zong and J. L. McHale. J. Chem. Phys., 106(12), 4963-4972, (1997).

10. A. R. Guerrero and R. F. Aroca, Angew. Chemie Int. Ed., 50(3), 665-668, (2011).

11. A. R. Guerrero, Y. Zhang, and R. F. Aroca. Small, 8(19), 2964-2967, (2012).

12. J. F. Li, Y. F. Huang, Y. Ding, Z. L. Yang, S. B. Li, X. S. Zhou, F. R. Fan, W. Zhang, Z. Y. Zhou, D. Y. Wu, B. Ren, Z. L. Wang and Z. Q. Tian. Nature, 464(7287), 392-395, (2010).

13. V. G. Machado, R. I. Stock, and C. Reichardt. Chem. Rev., 114(20), 10429-10475, (2014).

14. P. Lee and D. Meisel. J. Phys. Chem., 86(17), 3391-3395, (1982).

15. E. C. Le Ru, E. Blackie, M. Meyer, and P. G. Etchegoin. J. Phys. Chem. C, 111(37), 13794-13803, (2007).

16. M. J. Frisch, G. W. Trucks, H. B. Schlegel, G. E. Scuseria, M. A. Robb, J. R. Cheeseman, J. J. A. Montgomery, T. Vreven, K. N. Kudin, J. C. Burant, J. M. Millam, S. S. Iyengar, J. Tomasi, V. Barone, B. Mennucci, M. Cossi, G. Scalmani, N. Rega, G. A. Petersson, H. Nakatsuji, M. Hada, M. Ehara, K. Toyota, R. Fukuda, J. Hasegawa, M. Ishida, T. Nakajima, Y. Honda, O. Kitao, H. Nakai, M. Klene, X. Li, J. E. Knox, H. P. Hratchian, J. B. Cross, V. Bakken, C. Adamo, J. Jaramillo, R. Gomperts, R. E. Stratmann, O. Yazyev, A. J. Austin, R. Cammi, C. Pomelli, J. W. Ochterski, P. Y. Ayala, K. Morokuma, G. A. Voth, P. Salvador, J. J. Dannenberg, V. G. Zakrzewski, S. Dapprich, A. D. Daniels, M. C. Strain, O. Farkas, D. K Malick, A. D. Rabuck, K. Raghavachari, J. B. Foresman, J. V. Ortiz, Q. Cui, A. G. Baboul, S. Clifford, J. Cioslowski, B. B. Stefanov, G. Liu, A. Liashenko, P. Piskorz, I. Komaromi, R. L. Martin, D. J. Fox, T. Keith, M. A. Al-Laham, C. Y. Peng, A. Nanayakkara, M. Challacombe, P. M. W. 
Gill, B. Johnson, W. Chen, M. W. Wong, C. Gonzalez, and J. A. Pople, Gaussian 03, Revision C.02, 1, Gaussian, Inc.: Wallingford, Gaussian Inc., Wallingford CT, (2004).

17. W. Pietro, M. Francl, W. Hehre, D. DeFrees, J. Pople, and J. Binkley. J. Am. Chem. Soc., 104(19), 5039-5048, (1982).

18. P. Hariharan and J. Pople. Theor. Chim. Acta, 28(3), 213-222, (1973).

19. C. Lee, W. Yang, and R. Parr. Phys. Rev. B, 37(2), 785-789, (1988).

20. A. D. Becke. J. Chem. Phys., 98(7), 5648-5652, (1993).

21. W. Collier. QCPE Bull., 13, 16502-165013, (1996).

22. A. Aliaga, I. Osorio-Román, P. Leyton, C. Garrido, J. Cárcamo, C. Caniulef, F. Celis, G. Díaz F., E. Clavijo, J. S. Gómez-Jeria, and M. M. Campos-Vallette. J. Raman Spectrosc., 40(2), 164-169, (2009).

23. A. Aliaga, I. Osorio-Roman, C. Garrido, P. Leyton, J. Cárcamo, E. Clavijo, J. Gómez-Jeria, G. Díaz, and M. Campos-Vallette. Vib. Spectrosc., 50(1), 131-135, (2009).

24. P. Leyton, J. Gomez-Jeria, S. Sanchez-Cortes, C. Domingo, and M. Campos-Vallette, J. Phys. Chem. B., 110(13), 6470-6474, 2006.

25. A. Aliaga, H. Ahumada, K. Sepulveda, J. Gomez-Jeria, C. Garrido, B. Weiss-Lopez, and M. Campos-Vallette, J. Phys. Chem. C., 115(10), 3982 3989, (2011).
26. Hypercube Inc.: $1115 \mathrm{NW}$ 4th Street, Gainesville, FL. 32601, USA, (2007).

27. R. Hoffmann, J. Chem. Phys., 39(6), 1397-1412, (1963).

28. D. Lin-Vien, N. Colthup, W. Fateley, and J. Grasselli, The handbook of infrared and Raman characteristic frequencies of organic molecules, 1st ed. Chichester, UK: John Wiley \& Sons, (1991).

29. G. Socrates, Infrared and Raman Characteristic Group Frequencies, Tables and Charts. John Wiley and Sons: Chichester, (2001).

30. A. Hamid Othman, Z. Zakaria, and S. Weng Ng. J. Crystallogr. Spectrosc. Res., 23(12), 921-923, (1993).

31. B. Gu, C. Ruan, and W. Wang. Appl. Spectrosc., 63(1), 98-102, (2009).

32. J. S. Kang, S. Y. Hwang, C. J. Lee, and M. S. Lee. Bull. Korean Chem. Soc., 23(11), 1604-1610, (2002).

33. M. Moskovits. J. Chem. Phys., 77(9), 4408-4416, (1982).

34. D. R. Lide, Handbook of Chemistry and Physics, 82nd ed.; CRC Press: Boca Raton, FL, USA, (2001).

35. G. Schkolnik, J. Salewski, D. Millo, I. Zebger, S. Franzen, and P. Hildebrandt. Int. J. Mol. Sci., 13(6), 7466-7482, (2012).

36. R. F. Aroca, G. Y. Teo, H. Mohan, A. R. Guerrero, P. Albella, and F. Moreno. J. Phys. Chem. C, 115(42), 20419-20424, (2011). 